\title{
Uncovering subtle nail involvement in lichen planus with dermoscopy: a prospective, controlled study
}

\author{
Ömer Faruk Elmas \\ Department of Dermatology and Venereology, Faculty of Medicine, Ahi Evran University, Kırşehir, Turkey \\ Adv Dermatol Allergol 2020; XXXVII (3): 396-400 \\ DOI: https://doi.org/10.5114/ada.2020.96298
}

\begin{abstract}
Introduction: There are very few studies investigating the dermoscopic aspect of nail involvement in lichen planus and these studies described dermoscopic features of only clinically visible nail involvement in lichen planus.

Aim: To reveal subtle dermoscopic nail findings in patients with lichen planus.

Material and methods: The study included 40 patients with lichen planus and 40 healthy volunteers. All fingernails of the patients and healthy volunteers were examined by a handheld dermoscope and the findings detected were recorded. The patients were grouped by age, gender, disease duration and extent of the disease. The statistical analysis was performed using Chi square test.

Results: $82.5 \%$ of the patients and $17.5 \%$ of the healthy volunteers showed at least one dermoscopic nail finding. The frequency of multiple splinter haemorrhage $(p<0.05)$, multiple leukonychia $(p<0.05)$, longitudinal erythronychia $(p<0.05)$, prominent hyponychial vascular structures $(p<0.05)$ and onycholysis $(p<0.05)$ observed in the patients group was statistically significant. The other findings included distal short longitudinal lines, onychorrhexis, solitary splinter haemorrhage and solitary punctate leukonychia and showed no statistically significant differences between the two groups.

Conclusions: To the best of our knowledge, this is the first study focusing on uncovering subtle nail involvement in lichen planus. The presence of dilated hyponychial vascular structures in lichen planus was first described in the present study. It can be concluded that the frequency of nail involvement in lichen planus is more than known. Dermoscopic imaging of the nails of all patients with lichen planus may help establish early diagnosis and treatment to avoid permanent nail damage.
\end{abstract}

Key words: dermoscopy, lichen planus, nail.

\section{Introduction}

Lichen planus (LP) is a pruritic eruption involving the skin, nails, hair and mucosa [1]. The exact etiopathogenesis of the disease is unknown but it is thought to be a cell-mediated immune response associated with environmental triggers [2]. Violaceous, polygonal, flat-topped itchy papules and plaques located on flexural surfaces of the extremities are typical presentations of the cutaneous LP. Mucous membrane involvement is also common and may be found in patients who do not show cutaneous involvement [1].

The prevalence of LP is reported to be $0.5-1 \%$ of the population [3]. No significant geographical variation and racial predispositions have been noted for the disease. Some recent studies have shown that adult females are affected more than adult males [4]. LP can occur at any age including in children but most of the patients are aged 30-60 years [5].

Nail involvement is usually a manifestation of disseminated LP and can be seen up to $10 \%$ of patients with LP [6]. It may also be the only finding of the disease [7]. Nail LP affects the fingernails more than the toenails. Longitudinal ridging, pitting, distal splitting, onychorrhexis, brown discoloration, onycholysis and trachyonychia are known manifestations of nail LP [1].

Dermoscopy may be helpful in evaluation of disease progress and prognosis as the manifestations of nail matrix, nail bed and perionychium involvement can be observed clearly with this technique [6]. Nail LP may have a very progressive course, so early diagnosis and proper treatment are important [1].

There are very few studies about dermoscopic findings of nail LP $[6,8]$.

Address for correspondence: Ömer Faruk Elmas MD, Department of Dermatology and Venereology, Faculty of Medicine, Ahi Evran University, Kırşehir 40000, Turkey, phone: +90 5330260679, e-mail: omerfarukmd@gmail.com Received: 23.10.2018, accepted: 5.11.2018. 


\section{Aim}

Here we aimed to reveal dermoscopic nail findings in patients with cutaneous and mucosal lichen planus regarding the importance of early diagnosis. To the best of our knowledge, the present study is the first study focused on demonstrating subtle nail involvement without clinically visible changes.

\section{Material and methods}

The study included 40 patients with histopathologically confirmed mucocutaneous lichen planus and 40 age- and gender-matched healthy volunteers. The patients with any dermatological and systemic disease which may affect nails were not included. The patients who had a history of systemic treatment during the last 3 years were also not included. All fingernails of the patients and healthy volunteers were examined by a polarized-light handheld dermoscope with 10-fold magnification (DermLite DL4; 3Gen, San Juan Capistrano, CA). Dermoscopic photographing was performed with a dermoscope-adopted camera phone with high resolution (iPhone 7 plus, Apple, California, USA). The images were obtained after $2 \times$ optical zoom. All of the dermoscopic images were reviewed and the findings identified were recorded. The patients grouped by age, gender, disease duration and extent of the disease (localized/generalized).

\section{Statistical analysis}

The statistical analysis was performed with $\chi^{2}$ test using SPSS pocket program (SPSS Inc., Chicago, IL). $P<0.05$ was considered as statistically significant. All the procedures followed were in accordance with the Helsinki Declaration and the study was approved by the local clinical research ethics committee.

\section{Results}

The mean age of the patients and healthy volunteers were $46 \pm 13$ and $44 \pm 14$ years, respectively. Eighteen (45\%) patients were male and 22 (55\%) were female. Twenty (50\%) healthy volunteers were male and 20 (50\%) were female. There was no statistically significant difference between patients and control groups regarding the distribution of age and gender. The mean disease duration was 27 months. Thirty-three (82.5\%) of the patients and 12 (17.5\%) of the healthy volunteers showed at least one dermoscopic nail finding. Thirtythree $(82.5 \%)$ patients had localized and 7 (17.5\%) patients had generalized mucocutaneous involvement. The dermoscopic findings detected are detailed in Table 1. The presence of longitudinal erythronychia $(p<0.05)$ (Figures $1 \mathrm{~A}, \mathrm{~B})$, multiple splinter haemorrhage $(p<0.05)$ (Figures $2 \mathrm{~A}, \mathrm{~B}$ ), prominent hyponychial vascular structures $(p<0.05)$ (Figures 2 B, 3 A), multiple leukonychia $(p<0.05)$ (Figure 4 A) and onycholysis $(p<0.05)$ (Figures $3 \mathrm{~B}, 4 \mathrm{~B}$ ) in the patients group was statistically significant. The other findings included distal short longitudinal lines, onychorrhexis, solitary splinter haemorrhage and solitary punctate leukonychia and showed no statistically significant difference between the two groups. There was no statistically significant difference between male and female patients regarding the number of the nail findings detected.

When the patients grouped by age into four groups as 16-24 years, 25-35 years, 36-44 years and $45-68$ years; the number of the nail findings in the $45-68$ years group were higher and statistically significant $(p<0.05)$ when compared with the other age groups. When the disease duration was grouped as 1-3 months, 4-12 months, 1324 months and 24-120 months; the number of the nail findings detected in the 24-120 months group was statistically significant $(p<0.05)$. When the patients were

Table 1. Dermoscopic findings detected in the patients and control groups

\begin{tabular}{|c|c|c|c|}
\hline Dermoscopic finding & $\begin{array}{l}\text { The patients group } \\
\qquad(n=40)\end{array}$ & $\begin{array}{l}\text { The control group } \\
\qquad(n=40)\end{array}$ & $P$-values \\
\hline Multiple splinter haemorrhage & $12(30 \%)$ & $3(7.5 \%)$ & $<0.05$ \\
\hline Multiple leukonychia & $11(27.5 \%)$ & $4(10 \%)$ & $<0.05$ \\
\hline Longitudinal erythronychia & $6(15 \%)$ & None & $<0.05$ \\
\hline Onycholysis & $6(15 \%)$ & $1(2.5 \%)$ & $<0.05$ \\
\hline Prominent hyponychial vascular structures & $7(17.5 \%)$ & $1(2.5 \%)$ & $<0.05$ \\
\hline Melanonychia & $3(7.5 \%)$ & None & $>0.05$ \\
\hline Onychomadesis & $1(2.5 \%)$ & None & $>0.05$ \\
\hline Distal splitting & $1(2.5 \%)$ & None & $>0.05$ \\
\hline Solitary splinter haemorrhage & $6(15 \%)$ & $6(15 \%)$ & $>0.05$ \\
\hline Solitary punctate leukonychia & $7(17.5 \%)$ & $8(20 \%)$ & $>0.05$ \\
\hline Onychorrhexis & $11(27.5 \%)$ & $8(20 \%)$ & $>0.05$ \\
\hline Distal short longitudinal white lines & $6(15 \%)$ & $4(10 \%)$ & $>0.05$ \\
\hline
\end{tabular}




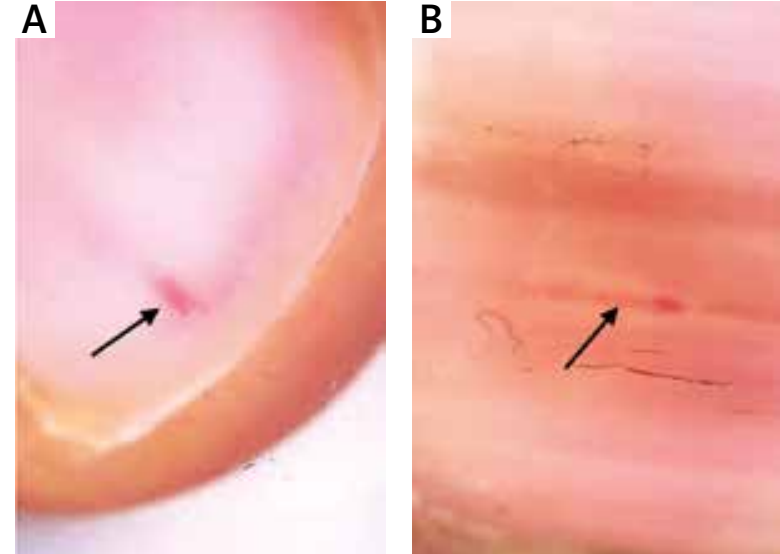

Figure 1. Longitudinal erythronychia (black arrow)
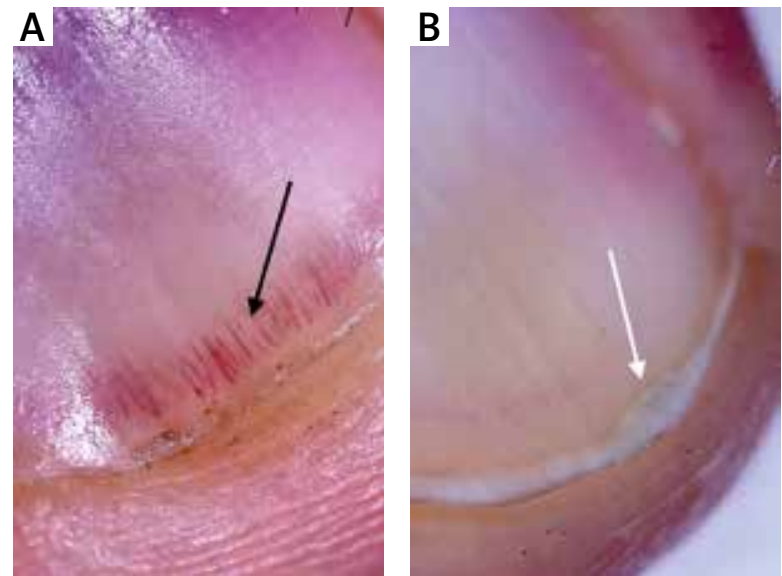

Figure 3. Prominent hyponychial vascular structures (black arrow) and onycholysis (white arrow)

grouped as localized $(n=32)$ and generalized $(n=8)$ mucocutaneous involvement, the number of the nail findings detected showed no statistically significant difference. In 5 (12.5\%) cases, only oral mucosa was involved. Mucocutaneous and only cutaneous involvement were seen in 22 (55\%) and 13 cases (32.5\%), respectively. Eighty percent of the cases with only oral mucosal involvement, $86 \%$ of the cases with mucocutaneous involvement and 77 cases with only cutaneous involvement showed dermoscopic nail involvement. There was no statistically significant difference between these three groups regarding the presence of dermoscopic findings.

\section{Discussion}

Nail LP shows variable progression including nail dystrophy, severe damage and even anonychia. So, early diagnosis is very important for proper management of the disease. Recently, dermoscopy of the nail, also known as
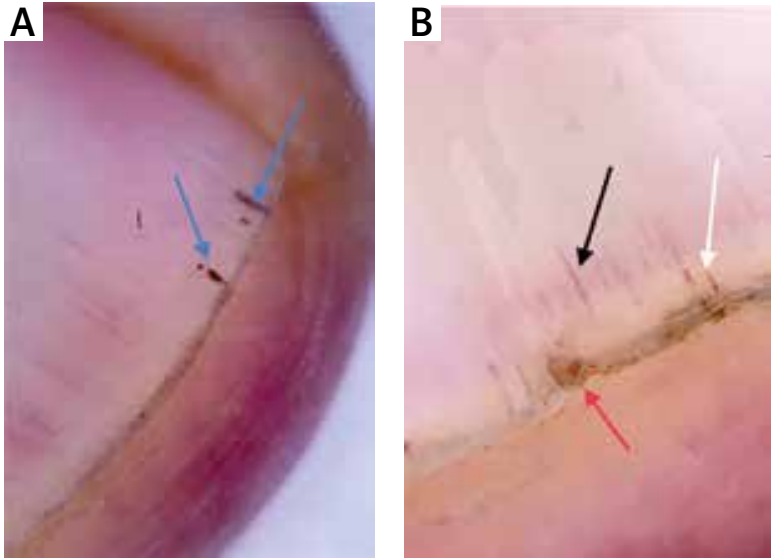

Figure 2. Splinter haemorrhage (blue arrows), prominent hyponychial vascular structures (black arrow), splinter haemorrhage (white arrow) and distal splitting (red arrow)
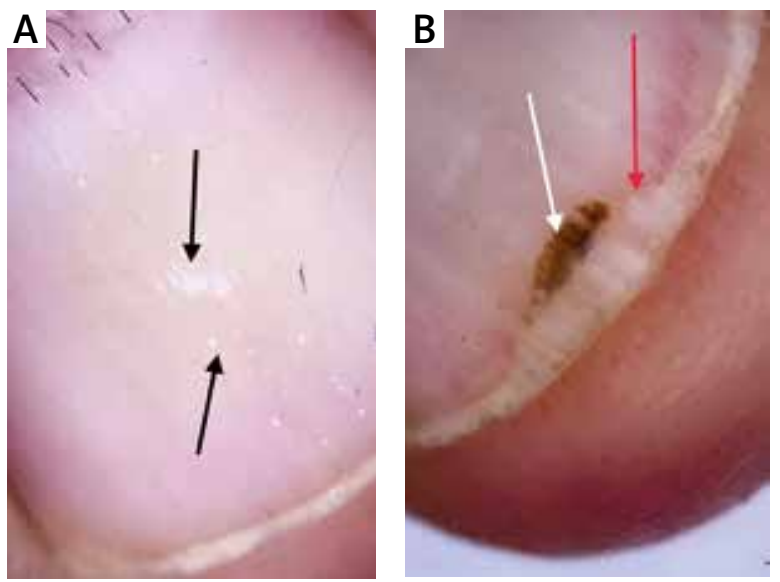

Figure 4. Punctate leukonychia (black arrows), melanonychia (white arrow), onycholysis (red arrow)

onychoscopy, has become an important tool providing early diagnosis in many non-neoplastic and neoplastic onychopathies including subungual hematoma, onychomycosis, nail psoriasis, melanonychia, subungual melanoma, onychopapilloma, onychomatricoma etc. [8].

To the best of our knowledge, there is just one study focusing on dermoscopic features of nail LP, in the literature. In this descriptive study, Nakamura et al. reviewed 79 affected nails of 11 patients with clinically visible nail LP [6]. Differently, here we focused on uncovering subtle and clinically invisible nail involvement in mucocutaneous LP to detect nail involvement as soon as possible.

When the literature is reviewed, it seems that the frequency of nail involvement has been reported in up to $10 \%$ of patients with $\operatorname{LP}[6,9,10]$. In the present study $25(60 \%)$ of the patients showed at least one statistically significant dermoscopic finding (at least one of the multiple splinter haemorrhage, multiple leukonychia, longitudinal erythronychia, prominence of hyponychial vas- 
cular structures and onycholysis). Sixteen (40\%) of these 25 patients had no clinically visible nail finding. So, it can be concluded that $40 \%$ of the patients showed a clinically invisible nail involvement.

Splinter haemorrhage can be described as tiny, linear, non-blanchable, reddish-brown to black, longitudinal streaks under the nail plate. SH of the nails can be seen in a wide variety of dermatological and internal diseases including psoriasis, lichen planus, vasculitis, connective tissue disorders, infectious diseases and renal failure [11]. In the study of Nakamura et al., the frequency of SH was found to be $35.44 \%$ of 11 cases with nail lichen planus [6]. In the present study, SH was considered in two groups as solitary (monodactylous) and multiple (polydactylous). The presence of solitary SH was not statistically significant between the patients and control group. However, $30 \%$ of the patients and $7.5 \%$ of the control group showed multiple $\mathrm{SH}$ and this difference was statistically significant $(p<0.05)$.

Leukonychia are small white areas in the nail plate caused by formation of deeper pits. It can be seen in different dermatological entities. Punctate leukonychia (PL) is usually found in nail plates of normal individuals [12]. Here we also considered PL in two groups as solitary and multiple. The presence of solitary PL was not statistically significant between the patients and control group. However, 27.55 of the patients and $10 \%$ of the control group showed multiple $\mathrm{PL}$ and this difference was statistically significant $(p<0.05)$.

Longitudinal erythronychia (LE) can be described as the presence of red bands in the nail unit and it can be monodactylous or polydactylous. The monodactylous form is usually caused by neoplastic conditions, however, the polydactylous form can occur in many disorders including LP, Darier's disease, amyloidosis, graft versus host disease, and epidermolysis bullosa [13]. In the present study, 6 (15\%) of the patients showed monodactylous LE. There was no dermoscopic finding which may be associated with a neoplastic process in these cases. LE was very subtle clinically in all of these 6 cases. In the study of Nakamura et al., none of the cases showed LE.

Onycholysis can be defined as separation of the nail plate from the nail bed. Onychomycosis, trauma and psoriasis are the main causes of onycholysis [14]. In our study, we detected onycholysis in 6 (15\%) patients and $1(2.5 \%)$ healthy volunteer and this difference was statistically significant $(p<0.05)$. In the study of Nakamura et al., $27.8 \%$ of the patients showed onycholysis.

Dilated hyponychial vascular structures can be seen in nail psoriasis and it was not described previously for LP [15]. We detected this finding in 7 (17.5\%) of the patients and one healthy volunteer. This difference was also statistically significant.

Onychorrhexis, also known as brittle nails, may be caused by physical factors like frequent soap and water exposure, nail polish remover and hypothyroidism [16].
Up to $20 \%$ of the population may be affected. In the present study, $27.5 \%$ of the patients and $20 \%$ of the healthy volunteers showed onychorrhexis. This difference was not significant statistically.

Onychomadesis is usually a late manifestation of the hand, foot and mouth disease. This finding was previously described for LP in a case report [17]. We also detected clinically evident onychomadesis in 1 patient.

Melanonychia is one of the previously reported clinical findings of nail lichen planus [18]. In our study, 7.5\% of the patients showed subtle melanonychia but this finding was not statistically significant.

The main limitation of our study was the lack of longterm follow-up of the patients with subtle nail involvement.

\section{Conclusions}

The novel aspects of the present study can be summarized as follows:

1. To the best of our knowledge, this is the first study focusing on uncovering subtle nail involvement in LP.

2. Statistically significant dermoscopic findings were detected in $40 \%$ of the patients. It can be concluded that the frequency of nail involvement in LP is more than known.

3. The presence of dilated hyponychial vascular structures in LP, to the best of our knowledge, was first described in the present study.

4. We described the second case of onychomadesis associated with LP.

5. Dermoscopic imaging of the nails of all patients with lichen planus may help establish early diagnosis and treatment to avoid permanent nail damage.

6. We suggest that short-term monitoring of the patients with subtle nail involvement may lead to more effective management of the disease.

\section{Conflict of interest}

The author declares no conflict of interest.

\section{References}

1. Weston G, Payette M. Update on lichen planus and its clinical variants. Int J Womens Dermatol 2015; 1: 140-9.

2. Lehman JS, Tollefson MM, Gibson LE. Lichen planus. Int J Dermatol 2009; 48: 682-94.

3. Pereira ALC, Azulay RD. Líquen plano. In: Dermatologia. Azulay RD, Azulay DR, Azulay-Abulafia L (eds.). Azulay, $5^{\text {th }}$ edn. Guanabara, RJ: Kogan SA, 2008; 124-7.

4. Kyriakis KP, Terzoudi S, Palamaras I, et al. Sex and age distribution of patients with lichen planus. J Eur Acad Dermatol Venereol 2006; 20: 625-6.

5. Balasubramaniam P, Ogboli M, Moss C. Lichen planus in children: review of 26 cases. Clin Exp Dermatol 2008; 33: 457-9.

6. Nakamura R, Broce AA, Palencia DP, et al. Dermatoscopy of nail lichen planus. Int J Dermatol 2013; 52: 684-7. 
7. Tosti A, Peluso AM, Fanti PA, Piraccini BM. Nail lichen planus: clinical and pathologic study of twenty-four patients. J Am Acad Dermatol 1993; 28: 724-30.

8. Nakamura RC, Costa MC. Dermatoscopic findings in the most frequent onychopathies: descriptive analysis of 500 cases. Int J Dermatol 2012; 51: 483-5.

9. Daoud MS, Pittelkow MR. Lichen planus. In: Fitzpatrick's Dermatology in General Medicine. $7^{\text {th }}$ edn. Vol. 1. Gilchrest BA, Paller AS, Wolff K, et al. (eds.). McGraw-Hill, New York, NY 2008; 244-55.

10. Baran R, Dawber R, Haneke E, Tosti A. Treatment in common nail disorders. In: A Text Atlas of Nail Disorders: Techniques in Investigation and Diagnosis. $3^{\text {rd }}$ edn. Tosti A, Baran R, Dawber R, et al. (eds.). Martin Dunitz, London 2003; 320-1.

11. Haber R, Khoury R, Kechichian E, Tomb R. Splinter hemorrhages of the nails: a systematic review of clinical features and associated conditions. Int I Dermatol 2016; 55: 1304-10.

12. Tüzün Y, Karakuş Ö. Leukonychia. J Turk Acad Dermatol 2009; 3.1: 93101r.

13. Scher RK. Evaluation of nail lines: color and shape hold clues. Cleve Clin J Med 2016; 83: 385-91.

14. Alessandrini A, Starace M, Piraccini BM. Dermoscopy in the evaluation of nail disorders. Skin Append Disord 2017; 3 : 70-82.

15. Yorulmaz A, Artuz F. A study of dermoscopic features of nai psoriasis. Adv Dermatol Allergol 2017; 34: 28-35.

16. Uyttendaele H, Geyer A, Scher RK. Brittle nails: pathogenesis and treatment. J Drugs Dermatol 2003; 2: 48-9.

17. Grover C, Vohra S. Onychomadesis with lichen planus: an under-recognized manifestation. Indian J Dermatol 2015; 60: 420 .

18. Juhlin L, Baran R. Longitudinal melanonychia after healing of lichen planus. Acta Derm Venereol 1989; 69: 338-9. 